\title{
MODELLING AND SIMULATION OF AN
}

\section{INDUCTION MACHINE}

\author{
Bhagyashree M.S. ${ }^{1}$, Mrs.Raksha Adappa ${ }^{2}$ \\ PG Scholar, NMAMIT, Nitte, India ${ }^{1}$ \\ Assistant Professor, NMAMIT, Nitte, India ${ }^{2}$
}

\begin{abstract}
Dynamic modelling approach is the easiest method used to describe an induction machine. Dynamic model gives information about transient behaviour of the system. In this paper, the dynamic model of the induction motor is represented in state space using Krause modelling equations. Synchronous reference frame is used to develop a model and flux equations are expressed in terms of reactance. The equations are grouped and for each group a subsystem is developed. Initially d-q modelling method is used for modelling of healthy induction machine. The result is simulated using MATLAB/SIMULINK.
\end{abstract}

Keywords: Induction Machine, d-q model, Flux Linkage, MATLAB/SIMULINK

\section{INTRODUCTION}

Induction machines are the commonly used machine in the industry because of its reliable operation, simple construction, low initial cost, easy operation, high efficiency, simple maintenance. Three phase induction machine is a self starting motor. It runs at constant speed from no load to full load. Compared to DC machine, the cost of induction machine per kVA is less and induction machine can be operated in hostile environment. Most electrical motor failure reduces production, interrupts a process and may damage other machineries related to motor. It is necessary to model a method which prevents unscheduled downtimes of induction machine. And also maintenance cost must be less.

Induction machine dynamic modelling and simulation is the most commonly used method in industry and academia. Dynamic modelling can be developed by writing differential equations. This type of modelling assumes that both stator and rotor windings are symmetric and MMF is sinusoidaly distributed. Equivalent resistance and inductance matrices are diagonal. This type of modelling is simple for computer simulation.

By establishing equivalence based on the equality of the MMF produced in the two phase and three phase windings and equal current magnitudes, dynamic model of the three phase induction machine is developed. Using Modular approach, it is easy to describe induction machine. Using this method, all of the machine parameters are easily accessible. Krause model is one of the most popular approaches. Study of Dynamic behaviour of induction machine has an effect upon the overall performance of the system. Using d-q transformation theory it is possible to get DC quantities from sinusoidal variable quantities and also, it is possible to control speed and torque of machine independently.

The induction machine dynamic model and equations used in this paper are from Bimal K.Bose, "Modern Power Electronics and AC Drives" [1] and R.Krishnan, "Electric motor drives: Modelling, analysis and Control" [5]. Lakhya Jyoti Phukon, Neelanjana Baruah[3] developed a generalized MATLAB SIMULINK model of a three phase induction motor. A model is tested for load and no load conditions. They presented that using s-function technique does not make use of the power of SIMULINK block. They concluded that using MATLAB/SIMULINK it is easy to predict and analyse the behaviour of an induction machine.

Burak Ozpineci and Leon M Tolbert [2] presented a paper on modular SIMULINK implementation of Induction machine model. They developed a subsystem block for each model equation. From this it is possible to get all of the machine parameters for control and verification. After the induction machine model, they presented a model for open loop constant $\mathrm{V} / \mathrm{Hz}$ control and indirect vector current.

\section{INDUCTION MOTOR MODEL}

To model an induction machine, the equations are obtained from dq0 equivalent circuit. The flux linkage model equations are as follows:

$$
\frac{\mathrm{dF}_{\mathrm{sq}}}{\mathrm{dt}}=\omega \mathrm{b}\left[\mathrm{v}_{\mathrm{sq}}-\frac{\omega_{\mathrm{e}}}{\omega_{\mathrm{b}}} \mathrm{F}_{\mathrm{sd}}+\frac{\mathrm{R}_{\mathrm{s}}}{\mathrm{x}_{\mathrm{sl}}}\left(\mathrm{F}_{\mathrm{qm}}+\mathrm{F}_{\mathrm{sq}}\right)\right]
$$




$$
\begin{gathered}
\text { NMAM Institute of Technology, Nitte } \\
\text { Vol. 4, Special Issue 2, April 2016 } \\
\frac{\mathrm{dF}_{\mathrm{sd}}}{\mathrm{dt}}=\omega \mathrm{b}\left[\mathrm{v}_{\mathrm{sd}}+\frac{\omega_{\mathrm{e}}}{\omega_{\mathrm{b}}} \mathrm{F}_{\mathrm{sd}}+\frac{\mathrm{R}_{\mathrm{s}}}{\mathrm{x}_{\mathrm{sl}}}\left(\mathrm{F}_{\mathrm{dm}}+\mathrm{F}_{\mathrm{sd}}\right)\right] \\
\frac{\mathrm{dF}_{\mathrm{rq}}}{\mathrm{dt}}=\omega_{\mathrm{b}}\left[\mathrm{V}_{\mathrm{rq}}-\frac{\left(\omega_{\mathrm{e}}-\omega_{\mathrm{r}}\right)}{\omega_{\mathrm{b}}} \mathrm{F}_{\mathrm{rd}}+\frac{\mathrm{R}_{\mathrm{r}}}{\mathrm{x}_{\mathrm{rl}}}\left(\mathrm{F}_{\mathrm{qm}}-\mathrm{F}_{\mathrm{rq}}\right)\right] \\
\frac{\mathrm{dF} \mathrm{rd}}{\mathrm{dt}}=\omega_{\mathrm{b}}\left[\mathrm{v}_{\mathrm{rd}}+\frac{\left(\omega_{\mathrm{e}}-\omega_{\mathrm{r}}\right)}{\omega_{\mathrm{b}}} \mathrm{F}_{\mathrm{rq}}+\frac{\mathrm{R}_{\mathrm{s}}}{\mathrm{x}_{\mathrm{sl}}}\left(\mathrm{F}_{\mathrm{qm}}+\mathrm{F}_{\mathrm{dr}}\right)\right]
\end{gathered}
$$

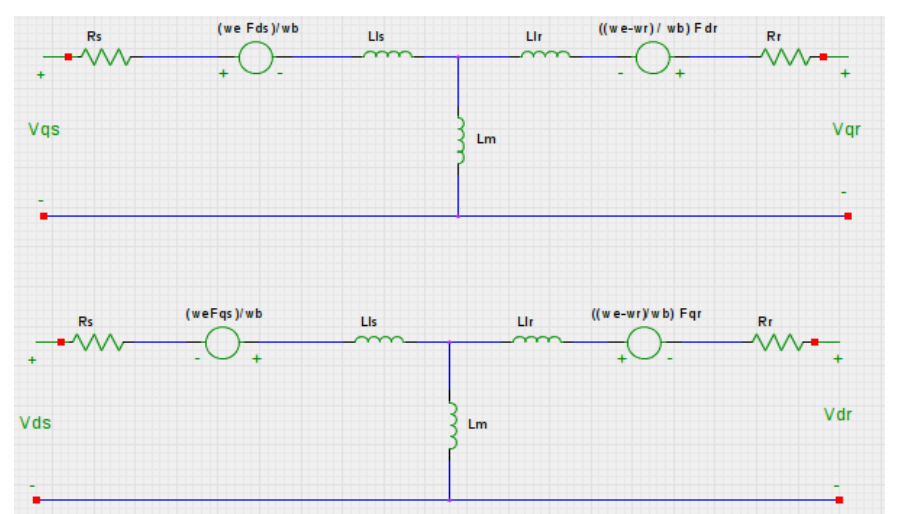

Figure 1: Synchronous frame DQ Equivalent Circuit of an Induction machine

$$
\begin{gathered}
\mathrm{F}_{\mathrm{qm}}=\mathrm{x}_{\mathrm{ml}} *\left[\frac{\mathrm{F}_{\mathrm{sq}}}{\mathrm{x}_{\mathrm{sl}}}+\frac{\mathrm{F}_{\mathrm{rq}}}{\mathrm{x}_{\mathrm{rl}}}\right] \\
\mathrm{F}_{\mathrm{dm}}=\mathrm{x}_{\mathrm{ml}} *\left[\frac{\mathrm{F}_{\mathrm{sd}}}{\mathrm{x}_{\mathrm{sl}}}+\frac{\mathrm{F}_{\mathrm{rd}}}{\mathrm{x}_{\mathrm{rl}}}\right] \\
\mathrm{i}_{\mathrm{sq}}=\frac{1}{\mathrm{x}_{\mathrm{sl}}}\left(\mathrm{F}_{\mathrm{sq}}-\mathrm{F}_{\mathrm{qm}}\right) \\
\mathrm{i}_{\mathrm{sd}}=\frac{1}{\mathrm{x}_{\mathrm{sl}}}\left(\mathrm{F}_{\mathrm{sd}}-\mathrm{F}_{\mathrm{dm}}\right) \\
\mathrm{i}_{\mathrm{rq}}=\frac{1}{\mathrm{x}_{\mathrm{rl}}}\left(\mathrm{F}_{\mathrm{rq}}-\mathrm{F}_{\mathrm{qm}}\right) \\
\mathrm{i}_{\mathrm{rd}}=\frac{1}{\mathrm{x}_{\mathrm{rl}}}\left(\mathrm{F}_{\mathrm{rd}}-\mathrm{F}_{\mathrm{dm}}\right) \\
\mathrm{T}_{\mathrm{e}}=\frac{3}{2}\left(\frac{\mathrm{P}}{2}\right) \frac{1}{\omega_{\mathrm{b}}}\left(\mathrm{F}_{\mathrm{sd}} \mathrm{i}_{\mathrm{sq}}-\mathrm{F}_{\mathrm{sq}} \mathrm{i}_{\mathrm{sd}}\right) \\
\mathrm{T}_{\mathrm{e}}-\mathrm{T}_{\mathrm{L}}=\left(\frac{2}{\mathrm{~J}}\right)\left(\frac{1}{\mathrm{P}}\right) \frac{\mathrm{d} \omega_{\mathrm{r}}}{\mathrm{dt}}
\end{gathered}
$$

where, $d$ is Direct Axis, $q$ is quadrature Axis, $s$ is Stator Variable, $r$ is Rotor Variable, $F_{\mathrm{ji}}$ : Flux Linkage $(\mathrm{j}=\mathrm{s}$ or $\mathrm{r}$ and $\mathrm{i}=\mathrm{q}$ or $\mathrm{d}$ ) $\mathrm{v}_{\mathrm{sq}}, \mathrm{v}_{\mathrm{sd}}$ : Stator voltages of $\mathrm{q}$ and $\mathrm{d}$ axis, $\mathrm{v}_{\mathrm{rq}}, \mathrm{v}_{\mathrm{sd}}$ : :otor voltages of $\mathrm{q}$ and $\mathrm{d}$ axis, $\mathrm{F}_{\mathrm{qm}}, \mathrm{F}_{\mathrm{dm}}: \mathrm{q}$ and $\mathrm{d}$ axis , $\mathrm{i}_{\mathrm{sq}}, \mathrm{i}_{\mathrm{sd}}$ : stator currents of $q$ and $d$-axis, $i_{s q}, i_{s d}$ :rotor currents of $q$ and d-axis, Te: Torque of Electrical output, TL: Torque of Load , $\omega_{\mathrm{e}}$ : Electrical Frequency of stator angle, $\omega_{\mathrm{b}}$ : Electrical base Frequency of motor angle, $\omega_{\mathrm{r}}$ :Electrical Frequency of rotor angle.

For an induction machine $\mathrm{v}_{\mathrm{qs}}$ and $\mathrm{v}_{\mathrm{ds}}$ are zero in equation (3) and (4). Before solving, differential equation are arranged in state space form by inserting equations $\mathrm{F}_{\mathrm{qm}}$ and $\mathrm{F}_{\mathrm{dm}}$ in Flux Linkage Equation and by rearranging then modelling equation in state-space is obtained as follows: 
NMAM Institute of Technology, Nitte

Vol. 4, Special Issue 2, April 2016

$$
\begin{aligned}
& \frac{\mathrm{dF}_{\mathrm{sq}}}{\mathrm{dt}}=\omega_{\mathrm{b}}\left[\mathrm{v}_{\mathrm{sq}}-\frac{\omega_{\mathrm{e}}}{\omega_{\mathrm{b}}} \mathrm{F}_{\mathrm{sd}}+\frac{\mathrm{R}_{\mathrm{s}}}{\mathrm{x}_{\mathrm{sl}}}\left(\frac{\mathrm{x}_{\mathrm{ml}}{ }^{*}}{\mathrm{x}_{\mathrm{rl}}} \mathrm{F}_{\mathrm{rq}}+\left(\frac{\mathrm{x}_{\mathrm{ml}}{ }^{*}}{\mathrm{x}_{\mathrm{sl}}}-1\right) \mathrm{F}_{\mathrm{sq}}\right)\right] \\
& \frac{\mathrm{dF}_{\mathrm{sd}}}{\mathrm{dt}}=\omega_{\mathrm{b}}\left[\mathrm{v}_{\mathrm{sd}}+\frac{\omega_{\mathrm{e}}}{\omega_{\mathrm{b}}} \mathrm{F}_{\mathrm{sq}}+\frac{\mathrm{R}_{\mathrm{s}}}{\mathrm{x}_{\mathrm{sl}}}\left(\frac{\mathrm{x}_{\mathrm{ml}}{ }^{*}}{\mathrm{x}_{\mathrm{rl}}} \mathrm{F}_{\mathrm{rd}}+\left(\frac{\mathrm{x}_{\mathrm{ml}}{ }^{*}}{\mathrm{x}_{\mathrm{sl}}}-1\right) \mathrm{F}_{\mathrm{sd}}\right)\right] \\
& \frac{\mathrm{dF}_{\mathrm{rq}}}{\mathrm{dt}}=\omega_{\mathrm{b}}\left[-\frac{\left(\omega_{\mathrm{e}}-\omega_{\mathrm{r}}\right)}{\omega_{\mathrm{b}}} \mathrm{F}_{\mathrm{rd}}+\frac{\mathrm{R}_{\mathrm{r}}}{\mathrm{x}_{\mathrm{rl}}}\left(\frac{\mathrm{x}_{\mathrm{ml}}{ }^{*}}{\mathrm{x}_{\mathrm{sl}}} \mathrm{F}_{\mathrm{sq}}+\left(\frac{\mathrm{x}_{\mathrm{ml}}{ }^{*}}{\mathrm{x}_{\mathrm{rl}}}-1\right) \mathrm{F}_{\mathrm{rq}}\right)\right] \\
& \frac{\mathrm{dF}_{\mathrm{rd}}}{\mathrm{dt}}=\omega_{\mathrm{b}}\left[\frac{\left(\omega_{\mathrm{e}}-\omega_{\mathrm{r}}\right)}{\omega_{\mathrm{b}}} \mathrm{F}_{\mathrm{rq}}+\frac{\mathrm{R}_{\mathrm{r}}}{\mathrm{x}_{\mathrm{rl}}}\left(\frac{\mathrm{x}_{\mathrm{ml}}{ }^{*}}{\mathrm{x}_{\mathrm{sl}}} \mathrm{F}_{\mathrm{sd}}+\left(\frac{\mathrm{x}_{\mathrm{ml}}{ }^{*}}{\mathrm{x}_{\mathrm{rl}}}-1\right) \mathrm{F}_{\mathrm{rd}}\right)\right] \\
& \frac{\mathrm{d} \omega_{\mathrm{r}}}{\mathrm{dt}}=\left(\frac{\mathrm{P}}{2 \mathrm{~J}}\right)\left(\mathrm{T}_{\mathrm{e}}-\mathrm{T}_{\mathrm{L}}\right)
\end{aligned}
$$

A. Supply voltage Equation

\section{SIMULINK IMPLEMENTATION}

$$
\begin{aligned}
& \mathrm{v}_{\mathrm{a}}=\mathrm{v}_{\mathrm{pm}} \sin \omega \mathrm{t} \\
& \mathrm{v}_{\mathrm{b}}=\mathrm{v}_{\mathrm{pm}} \sin \left(\mathrm{wt}-\frac{2 \pi}{3}\right) \\
& \mathrm{v}_{\mathrm{c}}=\mathrm{v}_{\mathrm{pm}} \sin \left(\mathrm{wt}+\frac{2 \pi}{3}\right)
\end{aligned}
$$

B. o-n Conversion block

o-n conversion block is used only in isolated neutral system. The transformation can be done as follows:

$$
\left[\begin{array}{l}
\mathrm{v}_{\mathrm{an}} \\
\mathrm{v}_{\mathrm{bn}} \\
\mathrm{v}_{\mathrm{cn}}
\end{array}\right]=\left[\begin{array}{ccc}
\frac{2}{3} & -\frac{1}{3} & -\frac{1}{3} \\
-\frac{1}{3} & \frac{2}{3} & -\frac{1}{3} \\
-\frac{1}{3} & -\frac{1}{3} & \frac{2}{3}
\end{array}\right]\left[\begin{array}{l}
\mathrm{v}_{\mathrm{a} 0} \\
\mathrm{v}_{\mathrm{b} 0} \\
\mathrm{v}_{\mathrm{c} 0}
\end{array}\right]
$$

C. Unit Vector calculation Equation

Unit Vectors $\sin \theta_{\mathrm{es}}$ and $\cos \theta_{\mathrm{es}}$ are used in abc-syn and syn-abc conversion blocks. $\theta_{\mathrm{es}}$ is the integration of input voltage frequency $\Omega$ e. Unit vector are calculated by taking sine and cosine of angle $\theta_{\mathrm{es}}$.

$$
\theta_{\mathrm{es}}=\int \omega_{\mathrm{e}} \mathrm{dt}
$$

D. Abc to syn calculation block

Using below equations three-phase voltages are converted to two-phase synchronous frame voltages.

$$
\begin{aligned}
{\left[\begin{array}{c}
\mathrm{v}_{\mathrm{sq}}^{\mathrm{s}} \\
\mathrm{v}^{\mathrm{s}}{ }_{\mathrm{sd}}
\end{array}\right] } & =\left[\begin{array}{ccc}
1 & 0 & 0 \\
0 & -\frac{1}{\sqrt{3}} & \frac{1}{\sqrt{3}}
\end{array}\right]\left[\begin{array}{c}
\mathrm{v}_{\mathrm{an}} \\
\mathrm{v}_{\mathrm{bn}} \\
\mathrm{v}_{\mathrm{cn}}
\end{array}\right] \\
\mathrm{v}_{\mathrm{sq}} & =\mathrm{v}^{\mathrm{s}}{ }_{\mathrm{sq}} \cos \theta_{\mathrm{e}}-\mathrm{v}^{\mathrm{s}}{ }_{\mathrm{sd}} \sin \theta_{\mathrm{e}} \\
\mathrm{v}_{\mathrm{sd}} & =\mathrm{v}^{\mathrm{s}}{ }_{\mathrm{sq}} \sin \theta_{\mathrm{e}}+\mathrm{v}_{\mathrm{sd}}^{\mathrm{s}} \cos \theta_{\mathrm{e}}
\end{aligned}
$$

E. Syn-abc conversion block

The two phase synchronous frame currents are converted to two phase stator reference frame and then to three phase current.

$$
\begin{aligned}
& \mathrm{i}^{\mathrm{s}}{ }_{\mathrm{sq}}=\mathrm{v}_{\mathrm{sq}} \cos \theta_{\mathrm{e}}+\mathrm{v}_{\mathrm{sd}} \sin \theta_{\mathrm{e}} \\
& \mathrm{i}_{\mathrm{sd}}^{\mathrm{s}}=-\mathrm{v}_{\mathrm{sq}} \sin \theta_{\mathrm{e}}+\mathrm{v}_{\mathrm{sd}} \cos \theta_{\mathrm{e}} \\
& {\left[\begin{array}{l}
\mathrm{i}_{\mathrm{a} 0} \\
\mathrm{i}_{\mathrm{b} 0} \\
\mathrm{i}_{\mathrm{c} 0}
\end{array}\right]=\left[\begin{array}{cc}
1 & 0 \\
-\frac{1}{2} & -\frac{\sqrt{3}}{2} \\
-\frac{1}{2} & \frac{\sqrt{3}}{2}
\end{array}\right]\left[\begin{array}{l}
\mathrm{i}_{\mathrm{sq}}^{\mathrm{s}} \\
\mathrm{i}_{\mathrm{sd}}^{\mathrm{s}}
\end{array}\right]}
\end{aligned}
$$




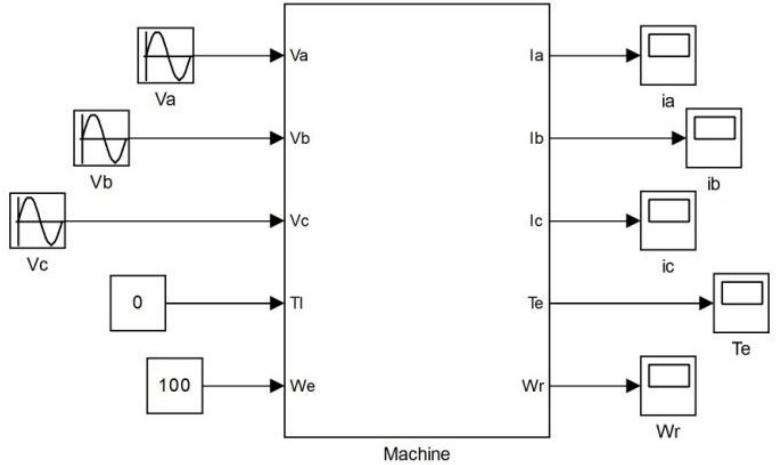

Figure 2: Induction Machine SIMULINK Model

IV.

RESULTS

The machine parameters have to be initialized before simulation by a m-file created in MATLAB. In this paper, a $30 \mathrm{KW}$ machine is modelled using MATLAB/SIMULINK. The input applied is $220 \mathrm{~V}$ and frequency is $60 \mathrm{~Hz}$. Initially, machine is tested at no load condition. I.e. by keeping load torque, $T_{L}=0$. The results show that the three phase currents speed and torque goes to steady state after some interval.
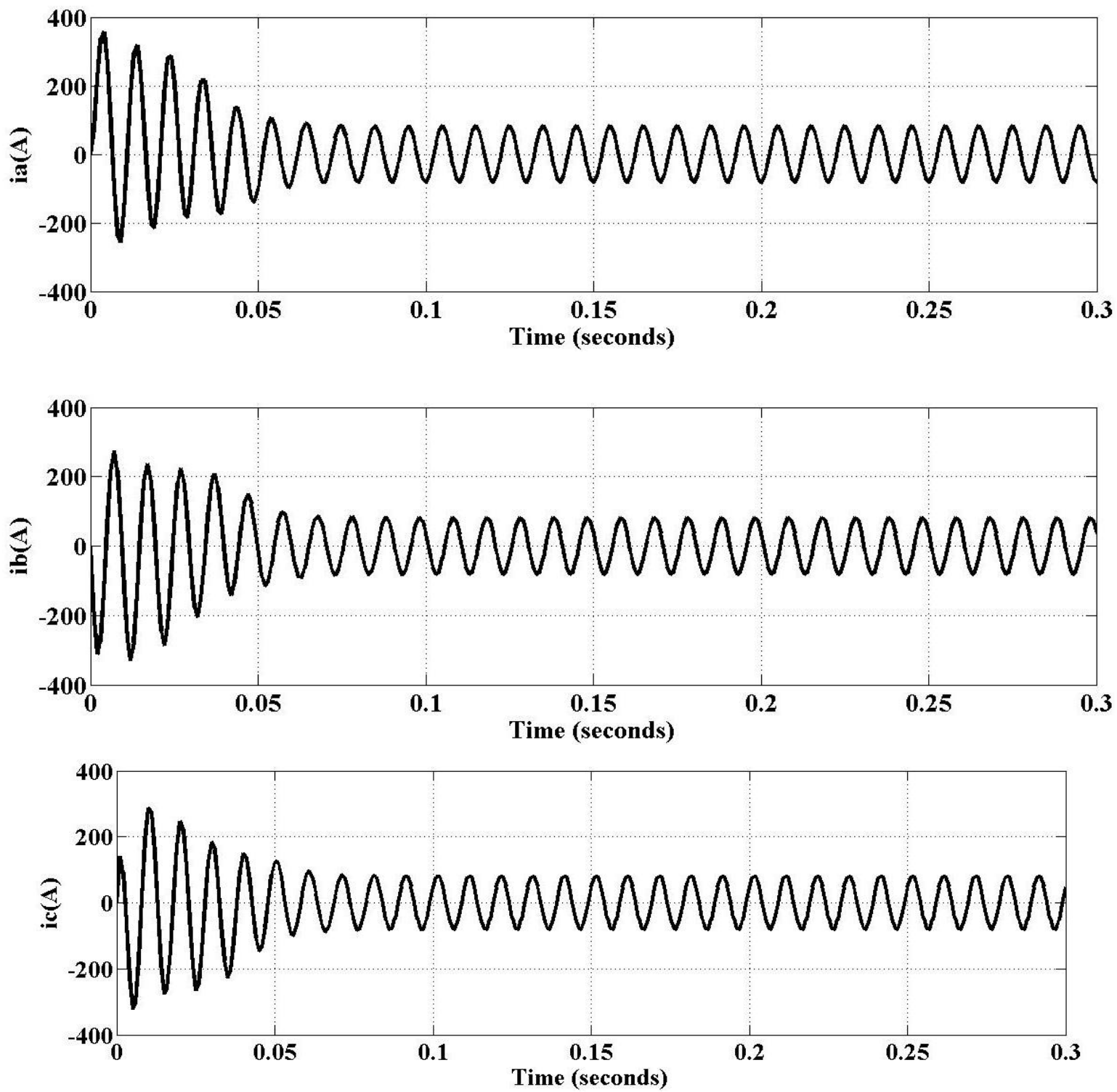

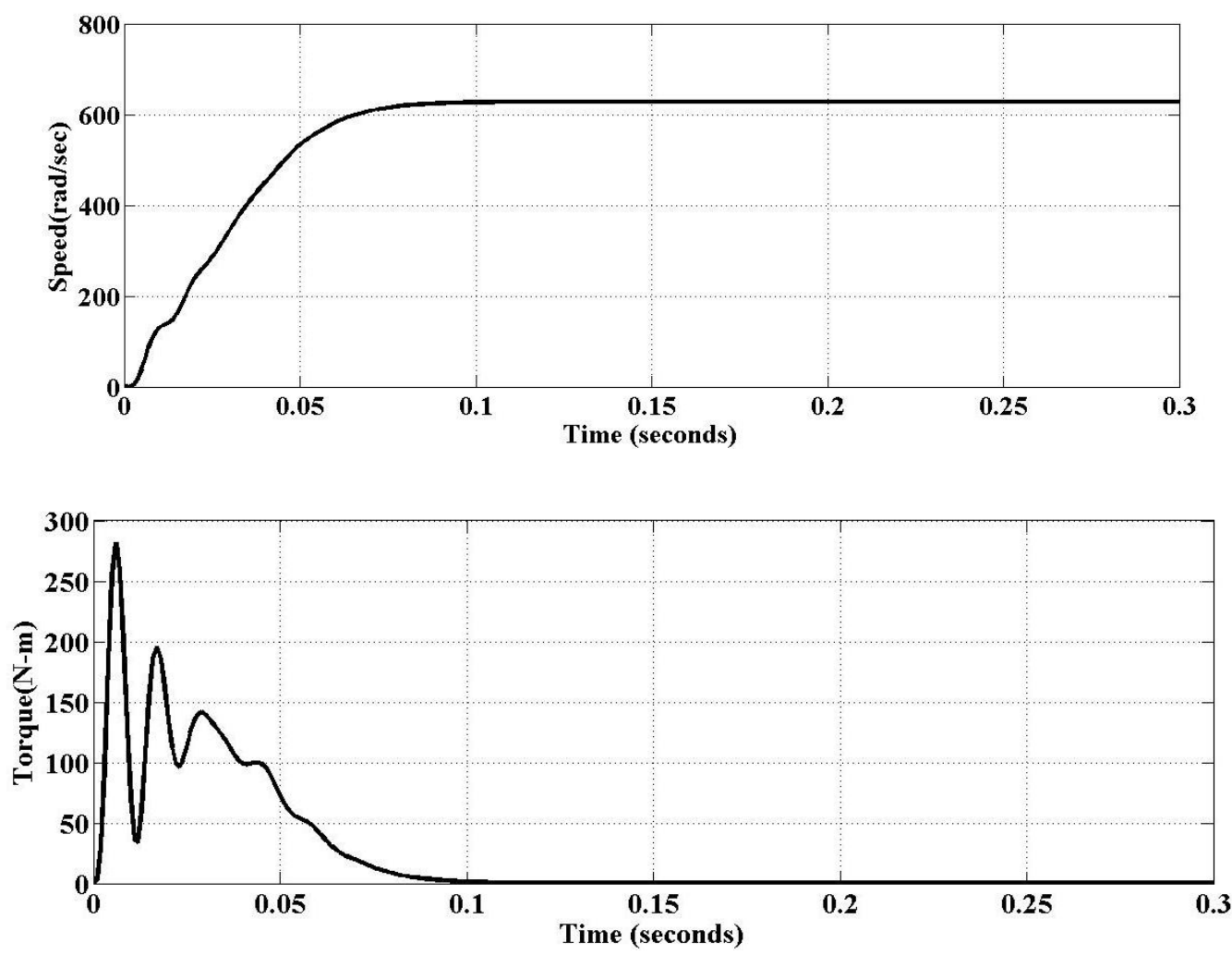

Figure 3: Simulation Results at no load condition

V.

CONCLUSION

In this paper, a $30 \mathrm{KW}$ induction machine is modelled using MATLAB/SIMULINK. The input applied is $220 \mathrm{~V}$ and frequency is $60 \mathrm{~Hz}$. Initially, machine is tested at no load condition. i.e. by keeping load torque, TL=0. The three phase current, speed and torque go to steady state after some interval. Here at $0.1 \mathrm{sec}$. As a value of speed increases, speed decreases.

A.Machine Parameters

\section{APPENDIX}

Rotor Resistance, $\mathrm{R}_{\mathrm{rr}}=0.39 \Omega$, Stator Resistance, $\mathrm{R}_{\mathrm{ss}}=0.19 \Omega$, Stator Inductance, Moment of Inertia,J=0.0226, $\mathrm{Lsl}=0.21 \mathrm{e}-3 \mathrm{H}$, Rotor Inductance, $\mathrm{Lrl}=0.6 \mathrm{e}-3 \mathrm{H}$, Magnetising Inductance, $\mathrm{Lm}=0.4 * \mathrm{e}-3 \mathrm{H}$, Base Frequency, fbb=100Hz, Number of Poles, $\mathrm{P}=4, \omega \mathrm{b}=2 * \mathrm{pi} * \mathrm{fbb}$, Stator Leakage Reactance, $\mathrm{Xsl}=\omega \mathrm{e} * \mathrm{Lsl}$, Rotor Leakage Reactance, Xrl $=\omega \mathrm{e} * \mathrm{Lrl}$ Magnetizing Impedence, $\mathrm{Xm}=\omega \mathrm{e} * \mathrm{Lm}, \mathrm{Xm} *=1 /(1 / \mathrm{Xls}+1 / \mathrm{Xm}+1 / \mathrm{Xlr})$

\section{ACKNOWLDEMENT}

I would like to thank my guide Mrs.Raksha Adappa, Assistant Professor, NMAMIT,Nitte. I sincerely express my gratitude to our Project Coordinator Mr.K.VASUDEVA SHETTIGAR Associate Professor and our HOD Dr.Nagesh Prabhu, Professor and Head, Department of E\&E, N.M.A.M Institute of Technology, Nitte for their timely scheduling of the project work and encouraging us to present papers.

\section{REFERENCES}

[1]. Bimal K. Bose,Power Electronics and Motor Drives-Advances and Trends, Academic Press,2006.

[2]. Burak Ozpineci, Leon M.Tolbert, "Simulink Implementation of Induction Machine Model-A Modular Approach", International Conference on Electric Machines and Drives(JEMD),Vol.2,pp.728-738,2003.

[3]. Lakhya Jyoti Phukon, Neelanjana Baruah, "A generalized Matlab Simulink Model of a Three Phase Induction Motor", International Journal of Innovative Research in Science, Engineering and Technology,Vol.4,Issue 5,May 2015.

[4]. P. Somasundaram. "A High Performance Induction Motor Drive System Using Fuzzy Logic Controller", TENCON 2006 - 2006 IEEE

[5]. Region 10 Conference, $11 / 2006$

[6]. R.Krishnan, Electric Motors Modelling Analysis and Control, Publication Prentice Hall of India,2002.

[7]. Samaga, B L Rajalakshmi, and K. P. Vittal. "Inclined mixed air gap eccentricity detection method for an induction motor", 2014, International Conference on Advances in Energy Conversion Technologies (ICAECT), 2014

[8]. Sushma, P., B.L. Rajalakshmi Samaga, and K.P. Vittal. "DQ modeling Of induction motor for virtual flux measurement", 2010 ,Conference Proceedings IPEC, 2010. 